CRYSTALLOGRAPHIC COMMUNICATIONS

ISSN 2056-9890

Received 7 November 2018

Accepted 15 November 2018

Edited by A. J. Lough, University of Toronto, Canada

Keywords: crystal structure; triazole; pyrimidine; hydrogen bond; $\pi \cdots \pi$-stacking; Hirshfeld surface analysis.

CCDC reference: 1879279

Supporting information: this article has supporting information at journals.iucr.org/e

\section{Crystal structure and Hirshfeld surface analysis of 5-methyl-1,2,4-triazolo[1,5-a]pyrimidine}

\author{
Sanae Lahmidi, ${ }^{\mathrm{a} *}$ Nada Kheira Sebbar, ${ }^{\mathrm{b}}$ Tuncer Hökelek, ${ }^{\mathrm{c}}$ Karim Chkirate, ${ }^{\mathrm{a}}$ Joel T. \\ Mague $^{d}$ and El Mokhtar Essassi ${ }^{\mathrm{a}}$
}

\begin{abstract}
a'Laboratoire de Chimie Organique Hétérocyclique URAC 21, Pôle de Compétence Pharmacochimie, Av. Ibn Battouta, BP 1014, Faculté des Sciences, Université Mohammed V, Rabat, Morocco, 'baboratoire de Chimie Bioorganique Appliquée, Faculté des Sciences, Université Ibn Zohr, Agadir, Morocco, ' Department of Physics, Hacettepe University, 06800 Beytepe, Ankara, Turkey, and d Department of Chemistry, Tulane University, New Orleans, LA 70118, USA. *Correspondence e-mail: sanaelahmidi2018@gmail.com
\end{abstract}

The nine-membered ring system of the title compound, $\mathrm{C}_{6} \mathrm{H}_{6} \mathrm{~N}_{4}$, is essentially planar. In the crystal, molecules are linked via $\mathrm{C}-\mathrm{H}_{\mathrm{Trz}} \cdots \mathrm{N}_{\mathrm{Trz}}$ and $\mathrm{C}-$ $\mathrm{H}_{\text {Pyrm }} \cdots \mathrm{N}_{\text {Trz }}(\mathrm{Trz}=$ triazole and Pyrm = pyrimidine $)$ hydrogen bonds together with weaker $\mathrm{C}-\mathrm{H}_{\mathrm{Pyrm}} \cdots \mathrm{N}_{\mathrm{Pyrm}}$ hydrogen bonds to form layers parallel to (102). The layers are further connected by $\pi-\pi$-stacking interactions between the ninemembered ring system [centroid-centroid = $3.7910(8) \AA$ ], forming oblique stacks along the $a$-axis direction. The Hirshfeld surface analysis of the crystal structure indicates that the most important contributions for the crystal packing are from $\mathrm{H} \cdots \mathrm{N} / \mathrm{N} \cdots \mathrm{H}(40.1 \%), \mathrm{H} \cdots \mathrm{H}(35.3 \%), \mathrm{H} \cdots \mathrm{C} / \mathrm{C} \cdots \mathrm{H}(9.5 \%), \mathrm{N} \cdots \mathrm{C} /$ $\mathrm{C} \cdots \mathrm{N}(9.0 \%), \mathrm{N} \cdots \mathrm{N}(3.1 \%)$ and $\mathrm{C} \cdots \mathrm{C}(3.0 \%)$ interactions and that hydrogenbonding and van der Waals interactions are the dominant interactions in the crystal packing. No significant $\mathrm{C}-\mathrm{H} \cdots \pi$ interactions are observed.

\section{Chemical context}

In recent years, much attention has been paid to the development of new methods for the synthesis and investigation of biological and pharmacological properties of $[1,2,4]$ triazolo[1,5-a]pyrimidine derivatives (Chebanov et al., 2010; Lahmidi et al., 2016a,b, 2018; Sedash et al., 2012). Thus, these compounds have also received successful applications for the preparation of new poly-condensed heterocycles (Beck et al., 2011). Among the various classes of nitrogen-containing heterocyclic compounds such as triazolopyrimidine derivatives display a broad spectrum of biological activities, including anti-inflammatory (Ashour et al., 2013), anticancer (Hoffmann et al., 2017) and antibacterial (Mabkhot et al., 2016) activities. In a continuation of our research on the elaboration of new methods for the synthesis of various heterocyclic systems, we investigated the reaction of bis(2chloroethyl)amine hydrochloride with ethyl 2-(5-methyl-11,2,4-triazolo[1,5-a]pyrimidin-7-yl)acetate under phasetransfer catalysis conditions using tetra- $n$-butyl ammoniumbromide (TBAB) as catalyst and potassium carbonate as base to afford the title compound, 5-methyl-1,2,4-triazolo[1,5-a]pyrimidine, (I). We report herein its molecular and crystal structures along with the results of a Hirshfeld surface analysis. 
<smiles>Cc1ccn2ncnc2n1</smiles>

\section{Structural commentary}

In the title compound (Fig. 1), the nine-membered ring is planar to within 0.004 (1) $\AA$ (for atom C5), and the r.m.s. deviation of the fitted atoms is $0.009 \AA$. Methyl atom C6 is displaced by 0.032 (1) $\AA$ from the ring system.

\section{Supramolecular features}

In the crystal, $\mathrm{C}-\mathrm{H}_{\mathrm{Trz}} \cdots \mathrm{N}_{\mathrm{Trz}}$ and $\mathrm{C}-\mathrm{H}_{\mathrm{Pyrm}} \cdots \mathrm{N}_{\mathrm{Trz}}(\mathrm{Trz}=$ triazole and Pyrm $=$ pyrimidine) hydrogen bonds (Table 1), together with weaker $\mathrm{C}-\mathrm{H}_{\text {Pyrm }} \cdots \mathrm{N}_{\text {Pyrm }}$ hydrogen bonds, link the molecules, forming layers parallel to (102) (Fig. 2). The layers are further connected by $\pi-\pi$-stacking interactions between the nine-membered rings [centroid-centroid distance $=3.7910(8) \AA]$, forming oblique stacks along the $a$-axis direction (Fig. 3). No significant $\mathrm{C}-\mathrm{H} \cdots \pi$ interactions are observed.

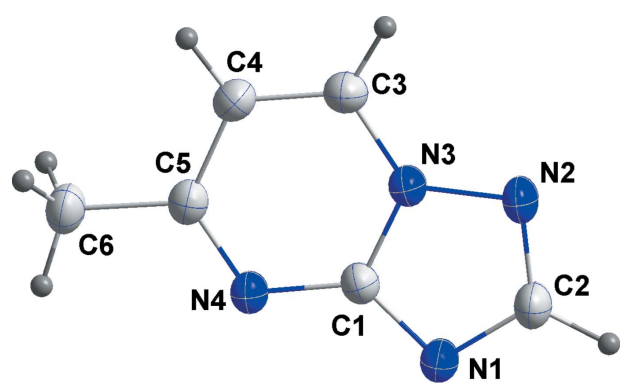

Figure 1

The title molecule with the atom-labelling scheme and $50 \%$ probability ellipsoids.

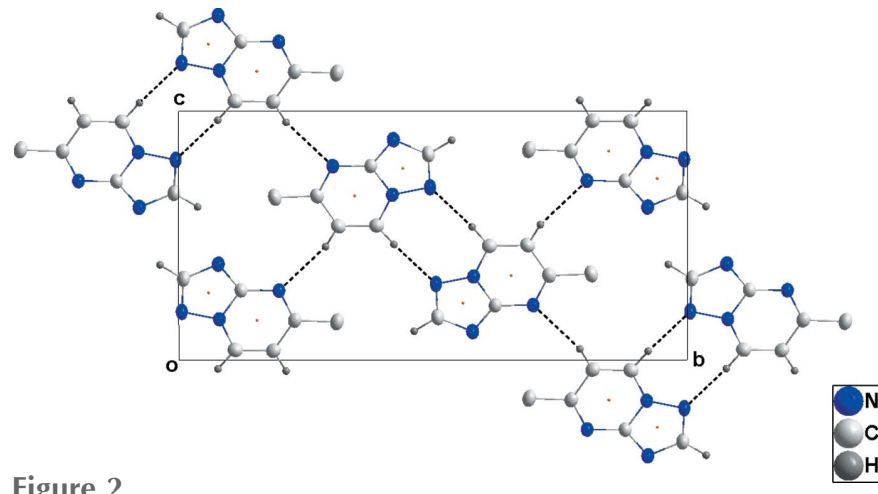

The packing viewed along the $a$-axis direction giving a plan view of the layers. $\mathrm{C}-\mathrm{H} \cdots \mathrm{N}$ hydrogen bonds are shown as black dashed lines and the orange dots mark the $\pi-\pi$ stacking interactions.
Table 1

Hydrogen-bond geometry $\left(\AA{ }^{\circ}\right)$.

\begin{tabular}{lllll}
\hline$D-\mathrm{H} \cdots A$ & $D-\mathrm{H}$ & $\mathrm{H} \cdots A$ & $D \cdots A$ & $D-\mathrm{H} \cdots A$ \\
\hline $\mathrm{C} 2-\mathrm{H} 2 \cdots \mathrm{N} 1^{\mathrm{i}}$ & $1.016(17)$ & $2.550(19)$ & $3.4052(18)$ & $141.5(13)$ \\
$\mathrm{C} 3-\mathrm{H} 3 \cdots \mathrm{N} 2^{\mathrm{vi}}$ & $0.979(18)$ & $2.525(18)$ & $3.4822(18)$ & $165.8(13)$ \\
$\mathrm{C} 4-\mathrm{H} 4 \cdots \mathrm{N} 4^{\mathrm{viii}}$ & $0.946(19)$ & $2.642(19)$ & $3.5677(17)$ & $165.9(14)$ \\
\hline $\begin{array}{l}\text { Symmetry codes: } \\
x-1,-y+\frac{1}{2}, z-\frac{1}{2} .\end{array}$ & (i) $-x+2,-y+1,-z+2 ;$ & $(\mathrm{vi})$ & $-x,-y+1,-z+1 ;$ & (viii)
\end{tabular}

\section{Hirshfeld surface analysis}

In order to visualize the intermolecular interactions in the crystal of the title compound, a Hirshfeld surface (HS) analysis (Hirshfeld, 1977; Spackman \& Jayatilaka, 2009) was carried out using CrystalExplorer17.5 (Turner et al., 2017). In the HS plotted over $d_{\text {norm }}$ (Fig. 4), the white surface indicates contacts with distances equal to the sum of the van der Waals

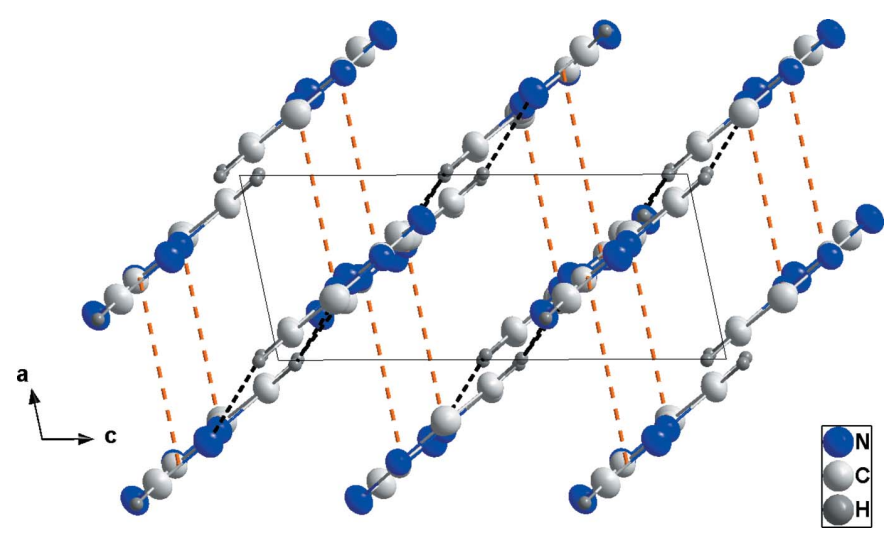

Figure 3

Packing seen along the $b$-axis direction giving a side view of the layers. Hydrogen bonds are depicted as in Fig. 2 and the $\pi$-stacking interactions are shown as orange dashed lines.

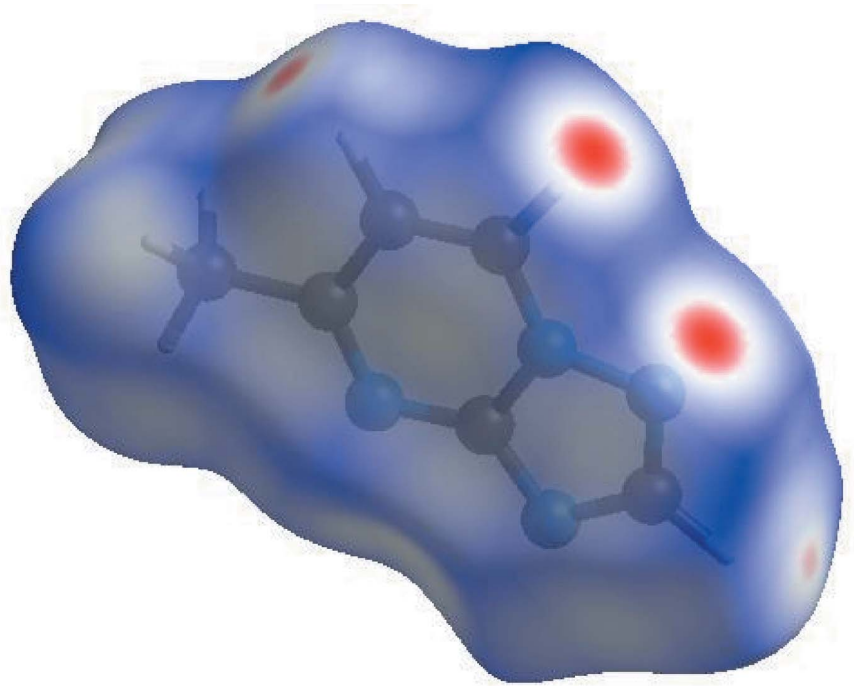

Figure 4

View of the three-dimensional Hirshfeld surface of the title compound plotted over $d_{\text {norm }}$ in the range -0.1566 to 1.0057 a.u. 


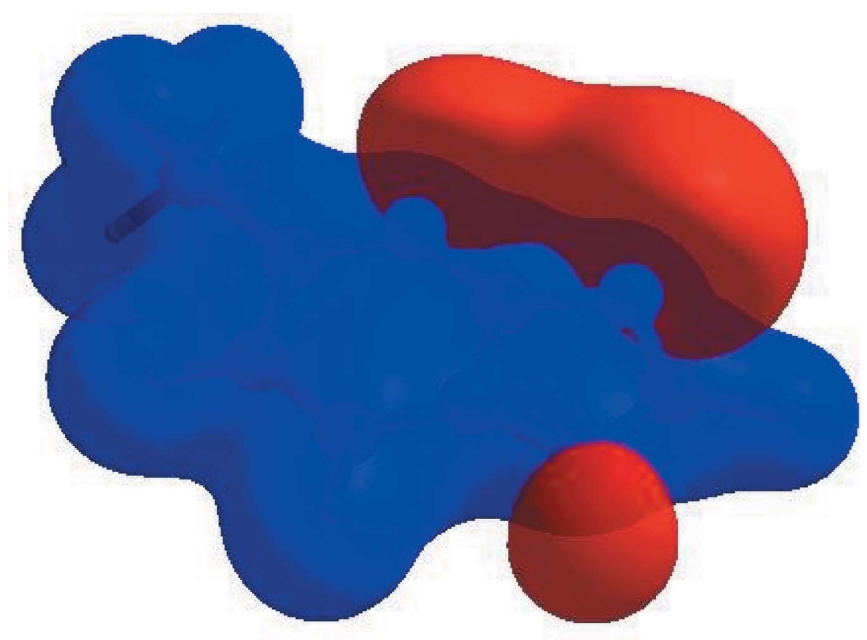

Figure 5

View of the three-dimensional Hirshfeld surface of the title compound plotted over electrostatic potential energy in the range -0.0500 to 0.0500 a.u. using the STO-3 G basis set at the Hartree-Fock level of theory. Hydrogen-bond donors and acceptors are shown as blue and red regions around the atoms corresponding to positive and negative potentials, respectively.

radii, and the red and blue colours indicate distances shorter (in close contact) or longer (distinct contact), respectively, than the van der Waals radii (Venkatesan et al., 2016). The bright-red spots appearing near N2 and hydrogen atoms $\mathrm{H} 2$, $\mathrm{H} 3$ and $\mathrm{H} 4$ indicate their roles as the respective donors and/or acceptors in the dominant $\mathrm{C}-\mathrm{H} \cdots \mathrm{N}$ hydrogen bonds; they also appear as blue and red regions corresponding to positive and negative potentials on the HS mapped over electrostatic potential (Spackman et al., 2008; Jayatilaka et al., 2005) as shown in Fig. 5. The blue regions indicate positive electrostatic potential (hydrogen-bond donors), while the red regions indicate negative electrostatic potential (hydrogen-bond acceptors). The shape-index of the HS is a tool to visualize $\pi-$ $\pi$ stacking by the presence of adjacent red and blue triangles;

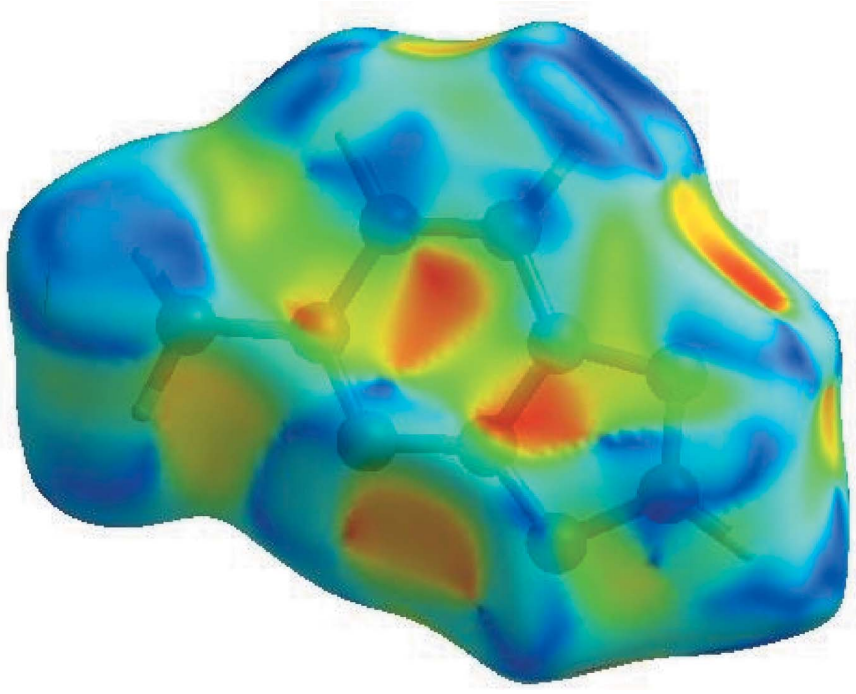

Figure 6

Hirshfeld surface of the title compound plotted over shape-index.
Table 2

Selected interatomic distances $(\AA)$.

\begin{tabular}{llll}
\hline $\mathrm{N} 1 \cdots \mathrm{C} 2^{\mathrm{i}}$ & $3.4051(19)$ & $\mathrm{C} 1 \cdots \mathrm{C} 4^{\text {iii }}$ & $3.5667(19)$ \\
$\mathrm{N} 2 \cdots \mathrm{C} 2^{\text {ii }}$ & $3.385(2)$ & $\mathrm{C} 2 \cdots \mathrm{C} 6^{\text {vii }}$ & $3.5715(18)$ \\
$\mathrm{N} 3 \cdots \mathrm{C} 3^{\text {iii }}$ & $3.4163(19)$ & $\mathrm{C} 2 \cdots \mathrm{C} 2^{\mathrm{i}}$ & $3.595(2)$ \\
$\mathrm{N} 4 \cdots \mathrm{C} 5^{\text {iii }}$ & $3.4314(17)$ & $\mathrm{C} 4 \cdots \mathrm{C} 5^{\text {ii }}$ & $3.4986(19)$ \\
$\mathrm{N} 4 \cdots \mathrm{C} 4^{\text {iii }}$ & $3.4177(19)$ & $\mathrm{C} 1 \cdots \mathrm{H} 6 B^{\text {iv }}$ & $2.94(3)$ \\
$\mathrm{N} 1 \cdots \mathrm{H} 6 B^{\text {iv }}$ & $2.85(2)$ & $\mathrm{C} 6 \cdots \mathrm{H} 6 C^{\text {iii }}$ & $2.98(3)$ \\
$\mathrm{N} 1 \cdots \mathrm{H} 2^{\mathrm{i}}$ & $2.553(18)$ & $\mathrm{H} 2 \cdots \mathrm{C} 6^{\mathrm{vii}}$ & $2.773(16)$ \\
$\mathrm{N} 1 \cdots \mathrm{H} 6 C^{\mathrm{v}}$ & $2.86(3)$ & $\mathrm{H} 2 \cdots \mathrm{H} 6 B^{\mathrm{vii}}$ & $2.58(3)$ \\
$\mathrm{N} 2 \cdots \mathrm{H} 3^{\mathrm{vi}}$ & $2.525(18)$ & $\mathrm{H} 2 \cdots \mathrm{H} 6 C^{\mathrm{vii}}$ & $2.48(3)$ \\
$\mathrm{N} 4 \cdots \mathrm{H} 4^{\mathrm{v}}$ & $2.641(18)$ & $\mathrm{H} 6 A \cdots \mathrm{H} 4^{\mathrm{v}}$ & $2.59(3)$ \\
$\mathrm{N} 4 \cdots \mathrm{H} 6 B^{\text {iv }}$ & $2.84(3)$ & $\mathrm{H} 6 B \cdots \mathrm{H} 6 C^{\mathrm{iii}}$ & $2.47(4)$ \\
$\mathrm{C} 1 \cdots \mathrm{C} 3^{\text {iii }}$ & $3.4166(19)$ & & \\
\hline
\end{tabular}

Symmetry codes: (i) $-x+2,-y+1,-z+2$; (ii) $x-1, y, z$; (iii) $x+1, y$, $z$; (iv) $x,-y+\frac{1}{2}, z+\frac{1}{2} ; \quad$ (v) $\quad x+1,-y+\frac{1}{2}, z+\frac{1}{2} ; \quad$ (vi) $\quad-x,-y+1,-z+1$; (vii) $-x+1, y+\frac{1}{2},-z+\frac{3}{2}$.

if there are no adjacent red and/or blue triangles, then there are no $\pi-\pi$ interactions. Fig. 6 clearly suggest that there are $\pi-$ $\pi$ interactions present in the crystal structure of (I).

The overall two-dimensional fingerprint plot, Fig. 7(a), and those delineated into $\mathrm{H} \cdots \mathrm{N} / \mathrm{N} \cdots \mathrm{H}, \mathrm{H} \cdots \mathrm{H}, \mathrm{H} \cdots \mathrm{C} / \mathrm{C} \cdots \mathrm{H}$, $\mathrm{N} \cdots \mathrm{C} / \mathrm{C} \cdots \mathrm{N}, \mathrm{N} \cdots \mathrm{N}$ and $\mathrm{C} \cdots \mathrm{C}$ contacts (McKinnon et al., 2007) are illustrated in Fig. $7(b)-(g)$, respectively, together with their relative contributions to the Hirshfeld surface. The most important interaction is $\mathrm{H} \cdots \mathrm{N} / \mathrm{N} \cdots \mathrm{H}$, contributing $40.1 \%$ to the overall crystal packing, which is reflected in Fig. 7(b) as a pair of characteristic wings with the tips at $d_{\mathrm{e}}+d_{\mathrm{i}}$ $=2.40 \AA$ arising from the $\mathrm{C}-\mathrm{H} \cdots \mathrm{N}$ hydrogen bonds (Table 1) as well as from the $\mathrm{H} \cdots \mathrm{N} / \mathrm{N} \cdots \mathrm{H}$ contacts (Table 3 ). The split
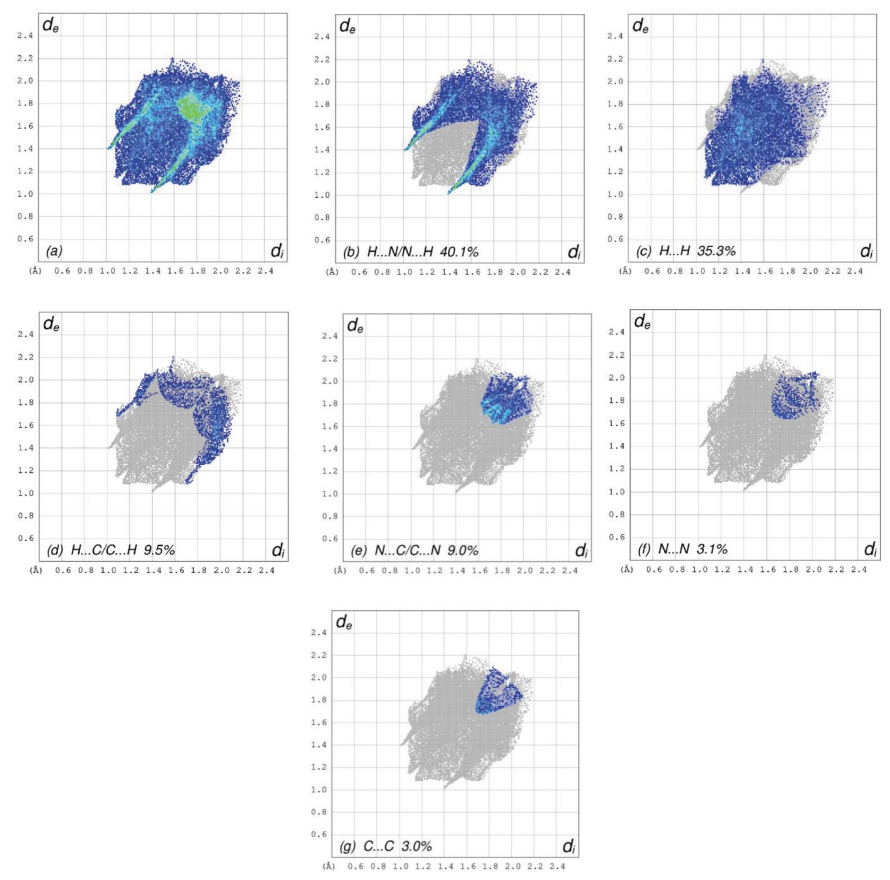

Figure 7

The full two-dimensional fingerprint plots for the title compound, showing $(a)$ all interactions, and delineated into $(b) \mathrm{H} \cdots \mathrm{N} / \mathrm{N} \cdots \mathrm{H},(c)$ $\mathrm{H} \cdots \mathrm{H},(d) \mathrm{H} \cdots \mathrm{C} / \mathrm{C} \cdots \mathrm{H},(e) \mathrm{N} \cdots \mathrm{C} / \mathrm{C} \cdots \mathrm{N},(f) \mathrm{N} \cdots \mathrm{N}$ and $(g) \mathrm{C} \cdots \mathrm{C}$ interactions. The $d_{\mathrm{i}}$ and $d_{\mathrm{e}}$ values are the closest internal and external distances (in $\AA$ ) from given points on the Hirshfeld surface contacts. 


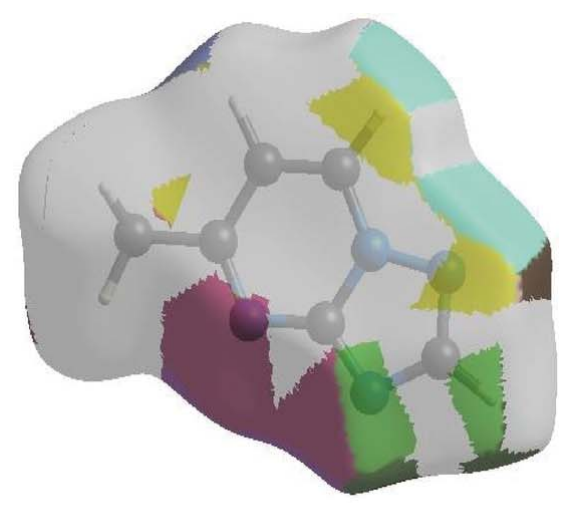

(a)

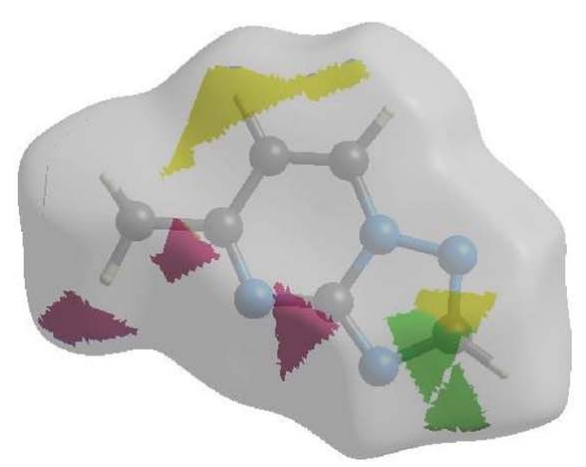

(c)

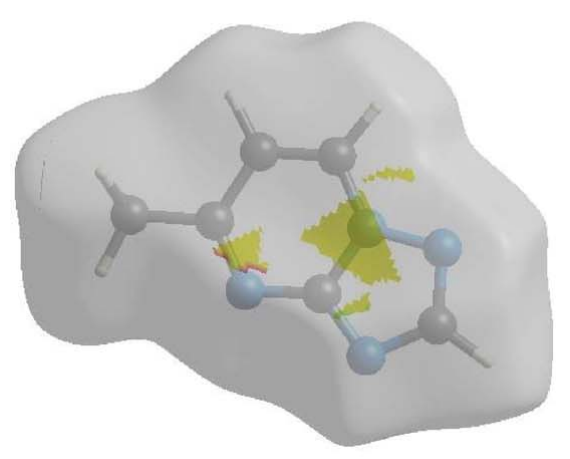

(e)

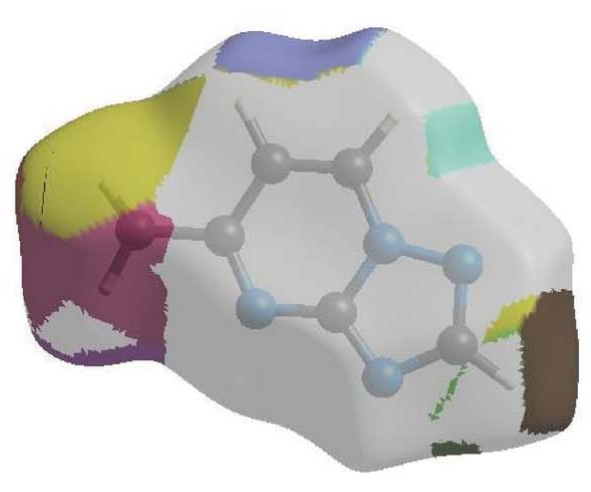

(b)

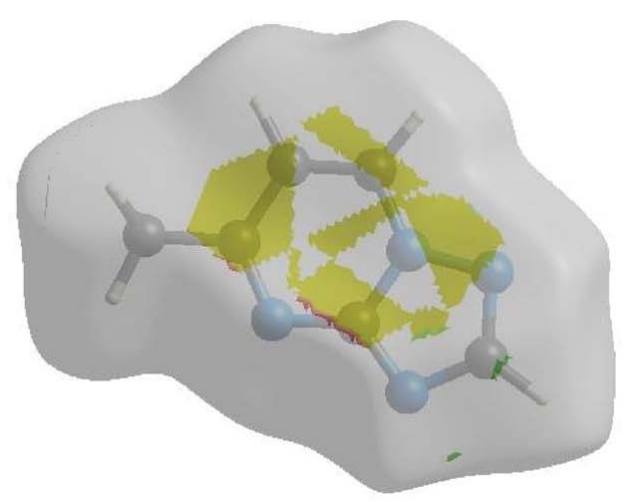

(d)

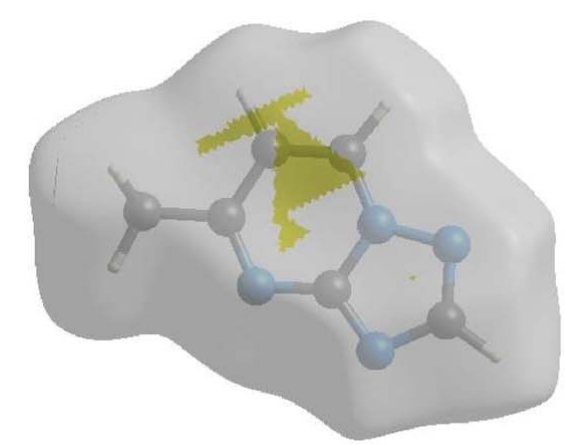

$(f)$

Figure 8

The Hirshfeld surface representations with the function $d_{\text {norm }}$ plotted onto the surface for $(a) \mathrm{H} \cdots \mathrm{N} / \mathrm{N} \cdots \mathrm{H},(b) \mathrm{H} \cdots \mathrm{H},(c) \mathrm{H} \cdots \mathrm{C} / \mathrm{C} \cdots \mathrm{H},(d) \mathrm{N} \cdots \mathrm{C} /$ $\mathrm{C} \cdots \mathrm{N},(e) \mathrm{N} \cdots \mathrm{N}$ and $(f) \mathrm{C} \cdots \mathrm{C}$ interactions.

thin and thick pair of wings with the tips at $d_{\mathrm{e}}+d_{\mathrm{i}} \sim 2.23 \AA$ in Fig. $7(c)$, arise from the short interatomic $\mathrm{H} \cdots \mathrm{H}$ contacts, which make a $35.3 \%$ contribution to the HS and are seen as widely scattered points of high density arising from the large hydrogen content of the molecule. In the absence of $\mathrm{C}-\mathrm{H} \cdots \pi$ interactions, the pair of wings in the fingerprint plot delineated into $\mathrm{H} \cdots \mathrm{C} / \mathrm{C} \cdots \mathrm{H}$ contacts $(9.5 \%$ contribution to the $\mathrm{HS})$ have a nearly symmetrical distribution of points, Fig. 7(d), with the tips at $d_{\mathrm{e}}+d_{\mathrm{i}} \sim 2.77 \AA$. The $\mathrm{N} \cdots \mathrm{C} / \mathrm{C} \cdots \mathrm{N}$ [Fig. 7(e)] and $\mathrm{N} \cdots \mathrm{N}[$ Fig. $7(f)]$ contacts make contributions of 9.0 and $3.1 \%$, respectively, to the HS and have widely scattered distributions of points. Finally, the C ‥C [Fig. $7(g)]$ contacts $(3.0 \%$ contribution to the HS) have a symmetrical distribution of points, with the tip at $d_{\mathrm{e}}=d_{\mathrm{i}}=1.69 \AA$.

The Hirshfeld surface representations with the function $d_{\text {norm }}$ plotted onto the surface are shown for the $\mathrm{H} \cdots \mathrm{N} / \mathrm{N} \cdots \mathrm{H}$, $\mathrm{H} \cdots \mathrm{H}, \mathrm{H} \cdots \mathrm{C} / \mathrm{C} \cdots \mathrm{H}, \mathrm{N} \cdots \mathrm{C} / \mathrm{C} \cdots \mathrm{N}, \mathrm{N} \cdots \mathrm{N}$ and $\mathrm{C} \cdots \mathrm{C}$ interactions in Fig. 8(a)-(f), respectively.

The Hirshfeld surface analysis confirms the importance of $\mathrm{H}$-atom contacts in establishing the packing. The large number of $\mathrm{H} \cdots \mathrm{N} / \mathrm{N} \cdots \mathrm{H}, \quad \mathrm{H} \cdots \mathrm{H}$ and $\mathrm{H} \cdots \mathrm{C} / \mathrm{C} \cdots \mathrm{H}$ interactions suggest that van der Waals interactions and hydrogen bonding play the major roles in the crystal packing (Hathwar et al., 2015). 
Table 3

Experimental details.

\begin{tabular}{|c|c|}
\hline \multicolumn{2}{|l|}{ Crystal data } \\
\hline Chemical formula & $\mathrm{C}_{6} \mathrm{H}_{6} \mathrm{~N}_{4}$ \\
\hline$M_{\mathrm{r}}$ & 134.15 \\
\hline Crystal system, space group & Monoclinic, $P 2_{1} / c$ \\
\hline Temperature (K) & 150 \\
\hline$a, b, c(\AA)$ & $3.7910(2), 18.0092(10), 9.0069(5)$ \\
\hline$\beta\left(^{\circ}\right)$ & $101.704(2)$ \\
\hline$V\left(\AA^{3}\right)$ & $602.14(6)$ \\
\hline$Z$ & 4 \\
\hline Radiation type & $\mathrm{Cu} K \alpha$ \\
\hline$\mu\left(\mathrm{mm}^{-1}\right)$ & 0.82 \\
\hline Crystal size (mm) & $0.29 \times 0.18 \times 0.13$ \\
\hline \multicolumn{2}{|l|}{ Data collection } \\
\hline Diffractometer & $\begin{array}{l}\text { Bruker D8 VENTURE PHOTON } \\
100 \text { CMOS }\end{array}$ \\
\hline Absorption correction & $\begin{array}{l}\text { Multi-scan (SADABS; Krause et } \\
\quad \text { al., 2015) }\end{array}$ \\
\hline$T_{\min }, T_{\max }$ & $0.67,0.90$ \\
\hline $\begin{array}{l}\text { No. of measured, independent and } \\
\text { observed }[I>2 \sigma(I)] \text { reflections }\end{array}$ & $4567,1205,1102$ \\
\hline$R_{\text {int }}$ & 0.074 \\
\hline$(\sin \theta / \lambda)_{\max }\left(\AA^{-1}\right)$ & 0.626 \\
\hline \multicolumn{2}{|l|}{ Refinement } \\
\hline$R\left[F^{2}>2 \sigma\left(F^{2}\right)\right], w R\left(F^{2}\right), S$ & $0.044,0.113,1.10$ \\
\hline No. of reflections & 1205 \\
\hline No. of parameters & 116 \\
\hline $\mathrm{H}$-atom treatment & All $\mathrm{H}$-atom parameters refined \\
\hline$\Delta \rho_{\max }, \Delta \rho_{\min }\left(\mathrm{e} \AA^{-3}\right)$ & $0.20,-0.20$ \\
\hline
\end{tabular}

Computer programs: APEX3 and SAINT (Bruker, 2016), SHELXT (Sheldrick, 2015a), SHELXL2018 (Sheldrick, 2015b), DIAMOND (Brandenburg \& Putz, 2012) and SHELXTL (Sheldrick, 2008).

\section{Database survey}

Two structures have previously been reported in which the title compound, (I), is present as a ligand $(L)$, namely $\left[\mathrm{Fe}(L)_{2}(\mathrm{SCN})_{2}\left(\mathrm{H}_{2} \mathrm{O}\right)_{2}\right]$ (Bigini Cingi et al., 1986) and $[\mathrm{Cu}(\mu-$ $\left.L)_{2}(\mathrm{SCN})\right]_{n}$ (Cornelissen et al., 1989), but to the best of our knowledge, the molecule itself has not previously been structurally characterized.

\section{Synthesis and crystallization}

To a solution of ethyl-2-\{5-methyl-1-[1,2,4]triazolo[1,5-a]pyrimidin-7-yl $\}$ acetate $(1.00 \mathrm{~g}, 4.5 \mathrm{mmol})$ in DMF $(25 \mathrm{ml})$ was added $2 \mathrm{eq}$ of bis(2-chloroethyl)amine hydrochloride $(1.61 \mathrm{~g}$, $9 \mathrm{mmol})$, potassium carbonate $(1.37 \mathrm{~g}, 9.9 \mathrm{mmol})$ and a catalytic amount of tetra- $n$-butylammonium bromide. The mixture was stirred at $353.15 \mathrm{~K}$ for $24 \mathrm{~h}$. The solution was filtered and the solvent was removed under reduced pressure. The residue obtained was dissolved in dichloromethane and purified by column chromatography (EtOAc/Hexane, 1:9 v:v). The title compound was obtained as colourless crystals in $40 \%$ yield.

\section{Refinement}

Crystal data, data collection and structure refinement details are summarized in Table 2 . $\mathrm{H}$ atoms were located in a difference Fourier map and were freely refined.

\section{Funding information}

The support of NSF-MRI grant No. 1228232 for the purchase of the diffractometer and Tulane University for support of the Tulane Crystallography Laboratory are gratefully acknowledged. TH is grateful to the Hacettepe University Scientific Research Project Unit (grant No. 013 D04 602 004).

\section{References}

Ashour, H., Shaaban, O., Rizk, O. \& El-Ashmawy, I. M. (2013). Eur. J. Med. Chem. 62, 341-351.

Beck, H. P., DeGraffenreid, M., Fox, B., Allen, J. G., Rew, Y., Schneider, S., Saiki, A. Y., Yu, D., Oliner, J. D., Salyers, K., Ye, Q. \& Olson, S. (2011). Bioorg. Med. Chem. Lett. 21, 2752-2755.

Biagini Cingi, M., Manotti Lanfredi, A. M., Tiripicchio, A., Cornelissen, J. P., Haasnoot, J. G. \& Reedijk, J. (1986). Acta Cryst. C42, 1296-1298.

Brandenburg, K. \& Putz, H. (2012). DIAMOND, Crystal Impact GbR, Bonn, Germany.

Bruker (2016). APEX3 and SAINT. Bruker AXS Inc., Madison, Wisconsin, USA.

Chebanov, V. A., Gura, K. A. \& Desenko, S. M. (2010). Top. Heterocycl. Chem. 23, 41-84.

Cornelissen, J. P., De Graaff, R. A. G., Haasnoot, J. G., Prins, R., Reedijk, J., Biagini-Cingi, M., Manotti-Lanfredi, A. M. \& Tiripicchio, A. (1989). Polyhedron, 8, 2313-2320.

Hathwar, V. R., Sist, M., Jørgensen, M. R. V., Mamakhel, A. H., Wang, X., Hoffmann, C. M., Sugimoto, K., Overgaard, J. \& Iversen, B. B. (2015). IUCrJ, 2, 563-574.

Hirshfeld, H. L. (1977). Theor. Chim. Acta, 44, 129-138.

Hoffmann, K., Wiśniewska, J., Wojtczak, A., Sitkowski, J., Denslow, A., Wietrzyk, J., Jakubowski, M. \& Łakomska, I. (2017). J. Inorg. Biochem. 172, 34-45.

Jayatilaka, D., Grimwood, D. J., Lee, A., Lemay, A., Russel, A. J., Taylor, C., Wolff, S. K., Cassam-Chenai, P. \& Whitton, A. (2005). TONTO - A System for Computational Chemistry. Available at: http://hirshfeldsurface.net/

Krause, L., Herbst-Irmer, R., Sheldrick, G. M. \& Stalke, D. (2015). J. Appl. Cryst. 48, 3-10.

Lahmidi, S., El Hafi, M., Moussaif, A., Benchidmi, M., Essassi, E. M. \& Mague, J. T. (2018). IUCrData, 3, x181280.

Lahmidi, S., Sebbar, N. K., Boulhaoua, M., Essassi, E. M., Mague, J. T. \& Zouihri, H. (2016a). IUCrData, 1, x160870.

Lahmidi, S., Sebbar, N. K., Harmaoui, A., Ouzidan, Y., Essassi, E. M. \& Mague, J. T. (2016b). IUCrData, 1, x161946.

Mabkhot, Y. N., Alatibi, F., El-Sayed, N. N. E., Kheder, N. A. \& AlShowiman, S. (2016). Molecules, 21, 1036-1045.

McKinnon, J. J., Jayatilaka, D. \& Spackman, M. A. (2007). Chem. Commun. pp. 3814.

Sedash, Y. V., Gorobets, N. Y., Chebanov, V. A., Konovalova, I. S., Shishkin, O. V. \& Desenko, S. M. (2012). RSC Adv. 2, 67196728.

Sheldrick, G. M. (2008). Acta Cryst. A64, 112-122.

Sheldrick, G. M. (2015a). Acta Cryst. A71, 3-8.

Sheldrick, G. M. (2015b). Acta Cryst. C71, 3-8.

Spackman, M. A. \& Jayatilaka, D. (2009). CrystEngComm, 11, 1932.

Spackman, M. A., McKinnon, J. J. \& Jayatilaka, D. (2008). CrystEngComm, 10, 377-388.

Turner, M. J., McKinnon, J. J., Wolff, S. K., Grimwood, D. J., Spackman, P. R., Jayatilaka, D. \& Spackman, M. A. (2017). CrystalExplorer17. The University of Western Australia.

Venkatesan, P., Thamotharan, S., Ilangovan, A., Liang, H. \& Sundius, T. (2016). Spectrochim. Acta Part A, 153, 625-636. 


\section{supporting information}

Acta Cryst. (2018). E74, 1833-1837 [https://doi.org/10.1107/S2056989018016225]

Crystal structure and Hirshfeld surface analysis of 5-methyl-1,2,4-triazolo[1,5a]pyrimidine

Sanae Lahmidi, Nada Kheira Sebbar, Tuncer Hökelek, Karim Chkirate, Joel T. Mague and El

Mokhtar Essassi

Computing details

Data collection: APEX3 (Bruker, 2016); cell refinement: SAINT (Bruker, 2016); data reduction: SAINT (Bruker, 2016); program(s) used to solve structure: SHELXT (Sheldrick, 2015a); program(s) used to refine structure: SHELXL2018 (Sheldrick, 2015b); molecular graphics: DIAMOND (Brandenburg \& Putz, 2012); software used to prepare material for publication: SHELXTL (Sheldrick, 2008).

5-Methyl-1,2,4-triazolo[1,5-a]pyrimidine

Crystal data

$\mathrm{C}_{6} \mathrm{H}_{6} \mathrm{~N}_{4}$

$M_{r}=134.15$

Monoclinic, $P 2_{1} / c$

$a=3.7910(2) \AA$

$b=18.0092(10) \AA$

$c=9.0069(5) \AA$

$\beta=101.704(2)^{\circ}$

$V=602.14(6) \AA^{3}$

$Z=4$

\section{Data collection}

Bruker D8 VENTURE PHOTON 100 CMOS diffractometer

Radiation source: INCOATEC I $\mu$ S micro-focus source

Mirror monochromator

Detector resolution: 10.4167 pixels $\mathrm{mm}^{-1}$

$\omega$ scans

Absorption correction: multi-scan

(SADABS; Krause et al., 2015)

\section{Refinement}

Refinement on $F^{2}$

Least-squares matrix: full

$R\left[F^{2}>2 \sigma\left(F^{2}\right)\right]=0.044$

$w R\left(F^{2}\right)=0.113$

$S=1.10$

1205 reflections

116 parameters

0 restraints
$F(000)=280$

$D_{\mathrm{x}}=1.480 \mathrm{Mg} \mathrm{m}^{-3}$

$\mathrm{Cu} K \alpha$ radiation, $\lambda=1.54178 \AA$

Cell parameters from 3969 reflections

$\theta=4.9-74.7^{\circ}$

$\mu=0.82 \mathrm{~mm}^{-1}$

$T=150 \mathrm{~K}$

Column, colourless

$0.29 \times 0.18 \times 0.13 \mathrm{~mm}$

$T_{\min }=0.67, T_{\max }=0.90$

4567 measured reflections

1205 independent reflections

1102 reflections with $I>2 \sigma(I)$

$R_{\text {int }}=0.074$

$\theta_{\max }=74.7^{\circ}, \theta_{\min }=4.9^{\circ}$

$h=-4 \rightarrow 4$

$k=-22 \rightarrow 21$

$l=-11 \rightarrow 10$

Primary atom site location: structure-invariant direct methods

Secondary atom site location: difference Fourier map

Hydrogen site location: difference Fourier map

All $\mathrm{H}$-atom parameters refined

$w=1 /\left[\sigma^{2}\left(F_{\mathrm{o}}^{2}\right)+(0.0458 P)^{2}+0.1643 P\right]$

where $P=\left(F_{\mathrm{o}}{ }^{2}+2 F_{\mathrm{c}}{ }^{2}\right) / 3$ 
$(\Delta / \sigma)_{\max }<0.001$

$\Delta \rho_{\max }=0.20 \mathrm{e} \AA^{-3}$

$\Delta \rho_{\min }=-0.20 \mathrm{e} \AA^{-3}$
Extinction correction: SHELXL2018 (Sheldrick, $2015 b), \mathrm{Fc}^{*}=\mathrm{kFc}\left[1+0.001 \mathrm{xFc}^{2} \lambda^{3} / \sin (2 \theta)\right]^{-1 / 4}$

Extinction coefficient: 0.021 (4)

\section{Special details}

Geometry. All esds (except the esd in the dihedral angle between two 1.s. planes) are estimated using the full covariance matrix. The cell esds are taken into account individually in the estimation of esds in distances, angles and torsion angles; correlations between esds in cell parameters are only used when they are defined by crystal symmetry. An approximate (isotropic) treatment of cell esds is used for estimating esds involving l.s. planes.

Refinement. Refinement of $\mathrm{F}^{2}$ against ALL reflections. The weighted R-factor $\mathrm{wR}$ and goodness of fit $\mathrm{S}$ are based on $\mathrm{F}^{2}$, conventional R-factors $R$ are based on $F$, with $F$ set to zero for negative $F^{2}$. The threshold expression of $F^{2}>2 \operatorname{sigma}\left(F^{2}\right)$ is used only for calculating R-factors(gt) etc. and is not relevant to the choice of reflections for refinement. R-factors based on $\mathrm{F}^{2}$ are statistically about twice as large as those based on F, and R- factors based on ALL data will be even larger.

Fractional atomic coordinates and isotropic or equivalent isotropic displacement parameters $\left(\AA^{2}\right)$

\begin{tabular}{lllll}
\hline & $x$ & $y$ & $z$ & $U_{\text {iso }} / U_{\text {eq }}$ \\
\hline $\mathrm{N} 1$ & $0.7561(3)$ & $0.42236(6)$ & $0.88570(13)$ & $0.0322(3)$ \\
$\mathrm{N} 2$ & $0.4407(3)$ & $0.49411(6)$ & $0.69436(15)$ & $0.0341(3)$ \\
$\mathrm{N} 3$ & $0.3764(3)$ & $0.42019(6)$ & $0.66430(13)$ & $0.0281(3)$ \\
$\mathrm{N} 4$ & $0.5532(3)$ & $0.30294(6)$ & $0.77958(13)$ & $0.0277(3)$ \\
$\mathrm{C} 1$ & $0.5676(3)$ & $0.37780(7)$ & $0.78059(15)$ & $0.0269(3)$ \\
$\mathrm{C} 2$ & $0.6678(4)$ & $0.49082(7)$ & $0.82759(17)$ & $0.0340(4)$ \\
$\mathrm{H} 2$ & $0.776(5)$ & $0.5371(9)$ & $0.883(2)$ & $0.037(4)^{*}$ \\
$\mathrm{C} 3$ & $0.1587(4)$ & $0.38918(7)$ & $0.54119(15)$ & $0.0313(3)$ \\
$\mathrm{H} 3$ & $0.025(5)$ & $0.4230(9)$ & $0.465(2)$ & $0.037(4)^{*}$ \\
$\mathrm{C} 4$ & $0.1409(4)$ & $0.31397(7)$ & $0.53851(16)$ & $0.0302(3)$ \\
$\mathrm{H} 4$ & $-0.006(5)$ & $0.2889(10)$ & $0.456(2)$ & $0.039(4)^{*}$ \\
$\mathrm{C} 5$ & $0.3442(3)$ & $0.27179(7)$ & $0.66009(15)$ & $0.0279(3)$ \\
$\mathrm{C} 6$ & $0.3279(4)$ & $0.18893(7)$ & $0.65408(19)$ & $0.0344(4)$ \\
$\mathrm{H} 6 \mathrm{~A}$ & $0.462(6)$ & $0.1656(13)$ & $0.749(3)$ & $0.066(6)^{*}$ \\
$\mathrm{H} 6 \mathrm{~B}$ & $0.431(6)$ & $0.1705(12)$ & $0.576(3)$ & $0.071(7)^{*}$ \\
$\mathrm{H} 6 \mathrm{C}$ & $0.095(7)$ & $0.1716(12)$ & $0.629(3)$ & $0.073(7)^{*}$ \\
& & & & \\
\hline
\end{tabular}

Atomic displacement parameters $\left(\AA^{2}\right)$

\begin{tabular}{lllllll}
\hline & $U^{11}$ & $U^{22}$ & $U^{33}$ & $U^{12}$ & $U^{13}$ & $U^{23}$ \\
\hline $\mathrm{N} 1$ & $0.0381(6)$ & $0.0233(5)$ & $0.0321(6)$ & $-0.0020(4)$ & $0.0000(5)$ & $-0.0010(4)$ \\
$\mathrm{N} 2$ & $0.0434(7)$ & $0.0197(5)$ & $0.0367(6)$ & $-0.0011(4)$ & $0.0026(5)$ & $0.0000(4)$ \\
$\mathrm{N} 3$ & $0.0324(6)$ & $0.0222(5)$ & $0.0281(6)$ & $-0.0001(4)$ & $0.0024(5)$ & $0.0005(4)$ \\
$\mathrm{N} 4$ & $0.0311(6)$ & $0.0221(5)$ & $0.0289(6)$ & $-0.0004(4)$ & $0.0040(5)$ & $-0.0003(4)$ \\
$\mathrm{C} 1$ & $0.0297(6)$ & $0.0224(6)$ & $0.0280(7)$ & $0.0001(4)$ & $0.0041(5)$ & $0.0011(4)$ \\
$\mathrm{C} 2$ & $0.0414(8)$ & $0.0221(6)$ & $0.0360(8)$ & $-0.0023(5)$ & $0.0020(6)$ & $-0.0019(5)$ \\
$\mathrm{C} 3$ & $0.0337(7)$ & $0.0304(7)$ & $0.0281(7)$ & $0.0005(5)$ & $0.0020(5)$ & $0.0005(5)$ \\
$\mathrm{C} 4$ & $0.0319(7)$ & $0.0289(7)$ & $0.0284(7)$ & $-0.0030(5)$ & $0.0026(5)$ & $-0.0029(5)$ \\
$\mathrm{C} 5$ & $0.0284(6)$ & $0.0250(6)$ & $0.0308(7)$ & $-0.0016(5)$ & $0.0074(5)$ & $-0.0022(5)$ \\
$\mathrm{C} 6$ & $0.0386(8)$ & $0.0244(7)$ & $0.0393(8)$ & $-0.0022(5)$ & $0.0058(7)$ & $-0.0043(5)$ \\
\hline
\end{tabular}


Geometric parameters $\left(\AA,{ }^{\circ}\right)$

\begin{tabular}{|c|c|c|c|}
\hline $\mathrm{N} 1-\mathrm{C} 1$ & $1.3329(17)$ & $\mathrm{C} 3-\mathrm{C} 4$ & $1.3560(18)$ \\
\hline $\mathrm{N} 1-\mathrm{C} 2$ & $1.3545(17)$ & $\mathrm{C} 3-\mathrm{H} 3$ & $0.979(18)$ \\
\hline $\mathrm{N} 2-\mathrm{C} 2$ & $1.329(2)$ & $\mathrm{C} 4-\mathrm{C} 5$ & $1.4241(19)$ \\
\hline $\mathrm{N} 2-\mathrm{N} 3$ & $1.3703(15)$ & $\mathrm{C} 4-\mathrm{H} 4$ & $0.946(19)$ \\
\hline $\mathrm{N} 3-\mathrm{C} 3$ & 1.3607 (17) & $\mathrm{C} 5-\mathrm{C} 6$ & $1.4940(18)$ \\
\hline $\mathrm{N} 3-\mathrm{C} 1$ & $1.3775(17)$ & C6-H6A & $1.00(2)$ \\
\hline $\mathrm{N} 4-\mathrm{C} 5$ & $1.3245(17)$ & $\mathrm{C} 6-\mathrm{H} 6 \mathrm{~B}$ & $0.94(3)$ \\
\hline $\mathrm{N} 4-\mathrm{C} 1$ & $1.3492(17)$ & $\mathrm{C} 6-\mathrm{H} 6 \mathrm{C}$ & $0.92(3)$ \\
\hline $\mathrm{C} 2-\mathrm{H} 2$ & $1.016(17)$ & & \\
\hline $\mathrm{N} 1 \cdots \mathrm{C} 2^{\mathrm{i}}$ & $3.4051(19)$ & $\mathrm{C} 1 \cdots \mathrm{C} 4^{\mathrm{iii}}$ & $3.5667(19)$ \\
\hline $\mathrm{N} 2 \cdots \mathrm{C} 2^{\mathrm{ii}}$ & $3.385(2)$ & $\mathrm{C} 2 \cdots \mathrm{C} 6^{\mathrm{vii}}$ & $3.5715(18)$ \\
\hline $\mathrm{N} 3 \cdots \mathrm{C} 3^{\mathrm{iii}}$ & $3.4163(19)$ & $\mathrm{C} 2 \cdots \mathrm{C} 2^{\mathrm{i}}$ & $3.595(2)$ \\
\hline $\mathrm{N} 4 \cdots \mathrm{C} 5^{\mathrm{iii}}$ & $3.4314(17)$ & $\mathrm{C} 4 \cdots \mathrm{C} 5^{\mathrm{ii}}$ & $3.4986(19)$ \\
\hline $\mathrm{N} 4 \cdots \mathrm{C} 4^{\mathrm{iii}}$ & 3.4177 (19) & $\mathrm{C} 1 \cdots \mathrm{H} 6 \mathrm{~B}^{\mathrm{iv}}$ & $2.94(3)$ \\
\hline $\mathrm{N} 1 \cdots \mathrm{H} 6 \mathrm{~B}^{\mathrm{iv}}$ & $2.85(2)$ & $\mathrm{C} 6 \cdots \mathrm{H} 6 \mathrm{C}^{\mathrm{iii}}$ & $2.98(3)$ \\
\hline $\mathrm{N} 1 \cdots \mathrm{H} 2^{\mathrm{i}}$ & $2.553(18)$ & $\mathrm{H} 2 \cdots \mathrm{C}^{\mathrm{vii}}$ & $2.773(16)$ \\
\hline $\mathrm{N} 1 \cdots \mathrm{H} 6 \mathrm{C}^{\mathrm{v}}$ & $2.86(3)$ & $\mathrm{H} 2 \cdots \mathrm{H} 6 \mathrm{~B}^{\mathrm{vii}}$ & $2.58(3)$ \\
\hline $\mathrm{N} 2 \cdots \mathrm{H} 3^{\mathrm{vi}}$ & $2.525(18)$ & $\mathrm{H} 2 \cdots \mathrm{H} 6 \mathrm{C}^{\mathrm{vii}}$ & $2.48(3)$ \\
\hline $\mathrm{N} 4 \cdots \mathrm{H} 4^{\mathrm{v}}$ & $2.641(18)$ & $\mathrm{H} 6 \mathrm{~A} \cdots \mathrm{H} 4^{\mathrm{v}}$ & $2.59(3)$ \\
\hline $\mathrm{N} 4 \cdots \mathrm{H} \mathrm{B}^{\mathrm{iv}}$ & $2.84(3)$ & H6B $\cdots H 6 C^{i i i}$ & $2.47(4)$ \\
\hline $\mathrm{C} 1 \cdots \mathrm{C} 3^{\mathrm{iii}}$ & $3.4166(19)$ & & \\
\hline $\mathrm{C} 1-\mathrm{N} 1-\mathrm{C} 2$ & $102.64(11)$ & $\mathrm{N} 3-\mathrm{C} 3-\mathrm{H} 3$ & $117.3(10)$ \\
\hline $\mathrm{C} 2-\mathrm{N} 2-\mathrm{N} 3$ & $101.05(10)$ & $\mathrm{C} 3-\mathrm{C} 4-\mathrm{C} 5$ & $120.13(12)$ \\
\hline $\mathrm{C} 3-\mathrm{N} 3-\mathrm{N} 2$ & $127.88(11)$ & $\mathrm{C} 3-\mathrm{C} 4-\mathrm{H} 4$ & $120.6(11)$ \\
\hline $\mathrm{C} 3-\mathrm{N} 3-\mathrm{C} 1$ & $122.05(11)$ & $\mathrm{C} 5-\mathrm{C} 4-\mathrm{H} 4$ & $119.3(11)$ \\
\hline $\mathrm{N} 2-\mathrm{N} 3-\mathrm{C} 1$ & $110.07(11)$ & $\mathrm{N} 4-\mathrm{C} 5-\mathrm{C} 4$ & $122.68(12)$ \\
\hline $\mathrm{C} 5-\mathrm{N} 4-\mathrm{C} 1$ & $116.45(11)$ & $\mathrm{N} 4-\mathrm{C} 5-\mathrm{C} 6$ & $117.78(12)$ \\
\hline $\mathrm{N} 1-\mathrm{C} 1-\mathrm{N} 4$ & $128.43(12)$ & $\mathrm{C} 4-\mathrm{C} 5-\mathrm{C} 6$ & $119.54(12)$ \\
\hline $\mathrm{N} 1-\mathrm{C} 1-\mathrm{N} 3$ & $109.26(11)$ & $\mathrm{C} 5-\mathrm{C} 6-\mathrm{H} 6 \mathrm{~A}$ & $112.4(14)$ \\
\hline $\mathrm{N} 4-\mathrm{C} 1-\mathrm{N} 3$ & $122.30(12)$ & $\mathrm{C} 5-\mathrm{C} 6-\mathrm{H} 6 \mathrm{~B}$ & $111.1(13)$ \\
\hline $\mathrm{N} 2-\mathrm{C} 2-\mathrm{N} 1$ & $116.97(12)$ & $\mathrm{H} 6 \mathrm{~A}-\mathrm{C} 6-\mathrm{H} 6 \mathrm{~B}$ & 106.4 (19) \\
\hline $\mathrm{N} 2-\mathrm{C} 2-\mathrm{H} 2$ & $122.2(10)$ & $\mathrm{C} 5-\mathrm{C} 6-\mathrm{H} 6 \mathrm{C}$ & $112.2(14)$ \\
\hline $\mathrm{N} 1-\mathrm{C} 2-\mathrm{H} 2$ & $120.8(10)$ & $\mathrm{H} 6 \mathrm{~A}-\mathrm{C} 6-\mathrm{H} 6 \mathrm{C}$ & $111.5(19)$ \\
\hline $\mathrm{C} 4-\mathrm{C} 3-\mathrm{N} 3$ & $116.38(12)$ & $\mathrm{H} 6 \mathrm{~B}-\mathrm{C} 6-\mathrm{H} 6 \mathrm{C}$ & $103(2)$ \\
\hline $\mathrm{C} 4-\mathrm{C} 3-\mathrm{H} 3$ & $126.3(10)$ & & \\
\hline $\mathrm{C} 2-\mathrm{N} 2-\mathrm{N} 3-\mathrm{C} 3$ & $179.68(13)$ & $\mathrm{N} 3-\mathrm{N} 2-\mathrm{C} 2-\mathrm{N} 1$ & $0.14(17)$ \\
\hline $\mathrm{C} 2-\mathrm{N} 2-\mathrm{N} 3-\mathrm{C} 1$ & $0.03(14)$ & $\mathrm{C} 1-\mathrm{N} 1-\mathrm{C} 2-\mathrm{N} 2$ & $-0.24(17)$ \\
\hline $\mathrm{C} 2-\mathrm{N} 1-\mathrm{C} 1-\mathrm{N} 4$ & $179.97(13)$ & $\mathrm{N} 2-\mathrm{N} 3-\mathrm{C} 3-\mathrm{C} 4$ & $-179.91(12)$ \\
\hline $\mathrm{C} 2-\mathrm{N} 1-\mathrm{C} 1-\mathrm{N} 3$ & $0.24(14)$ & $\mathrm{C} 1-\mathrm{N} 3-\mathrm{C} 3-\mathrm{C} 4$ & $-0.30(18)$ \\
\hline $\mathrm{C} 5-\mathrm{N} 4-\mathrm{C} 1-\mathrm{N} 1$ & $-179.67(12)$ & $\mathrm{N} 3-\mathrm{C} 3-\mathrm{C} 4-\mathrm{C} 5$ & $-0.18(19)$ \\
\hline $\mathrm{C} 5-\mathrm{N} 4-\mathrm{C} 1-\mathrm{N} 3$ & $0.04(17)$ & $\mathrm{C} 1-\mathrm{N} 4-\mathrm{C} 5-\mathrm{C} 4$ & $-0.53(17)$ \\
\hline $\mathrm{C} 3-\mathrm{N} 3-\mathrm{C} 1-\mathrm{N} 1$ & $-179.85(12)$ & $\mathrm{C} 1-\mathrm{N} 4-\mathrm{C} 5-\mathrm{C} 6$ & $178.84(11)$ \\
\hline $\mathrm{N} 2-\mathrm{N} 3-\mathrm{C} 1-\mathrm{N} 1$ & $-0.18(14)$ & $\mathrm{C} 3-\mathrm{C} 4-\mathrm{C} 5-\mathrm{N} 4$ & $0.6(2)$ \\
\hline
\end{tabular}




\begin{tabular}{llll}
$\mathrm{C} 3-\mathrm{N} 3-\mathrm{C} 1-\mathrm{N} 4$ & $0.39(18)$ & $\mathrm{C} 3-\mathrm{C} 4-\mathrm{C} 5-\mathrm{C} 6$ & $-178.74(13)$ \\
$\mathrm{N} 2-\mathrm{N} 3-\mathrm{C} 1-\mathrm{N} 4$ & $-179.93(11)$ & & \\
\hline
\end{tabular}

Symmetry codes: (i) $-x+2,-y+1,-z+2$; (ii) $x-1, y, z$; (iii) $x+1, y, z$; (iv) $x,-y+1 / 2, z+1 / 2$; (v) $x+1,-y+1 / 2, z+1 / 2$; (vi) $-x,-y+1,-z+1$; (vii) $-x+1, y+1 / 2$, $-z+3 / 2$.

Hydrogen-bond geometry $\left(\AA,{ }^{\circ}\right)$

\begin{tabular}{lllll}
\hline$D-\mathrm{H} \cdots A$ & $D-\mathrm{H}$ & $\mathrm{H} \cdots A$ & $D \cdots A$ & $D-\mathrm{H}^{\cdots} \cdots A$ \\
\hline $\mathrm{C} 2-\mathrm{H} 2 \cdots \mathrm{N} 1^{\mathrm{i}}$ & $1.016(17)$ & $2.550(19)$ & $3.4052(18)$ & $141.5(13)$ \\
$\mathrm{C} 3-\mathrm{H} 3 \cdots \mathrm{N} 2^{\mathrm{vi}}$ & $0.979(18)$ & $2.525(18)$ & $3.4822(18)$ & $165.8(13)$ \\
$\mathrm{C} 4-\mathrm{H} 4 \cdots \mathrm{N} 4^{\text {viii }}$ & $0.946(19)$ & $2.642(19)$ & $3.5677(17)$ & $165.9(14)$ \\
\hline
\end{tabular}

Symmetry codes: (i) $-x+2,-y+1,-z+2$; (vi) $-x,-y+1,-z+1$; (viii) $x-1,-y+1 / 2, z-1 / 2$. 